\title{
The Extension of Rod-Coil Multiblock Copolymers and the Effect of the Helix-Coil Transition
}

\author{
A. Buhot and A. Halperin \\ UMR 5819 (CEA, CNRS, UJF), DRFMC/SI3M, CEA-Grenoble, 17 rue des Martyrs, \\ 38054 Grenoble Cedex 9, France
}

(August 28, 2018)

\begin{abstract}
The extension elasticity of rod-coil mutliblock copolymers is analyzed for two experimentally accessible situations. In the quenched case, when the architecture is fixed by the synthesis, the force law is distinguished by a sharp change in the slope. In the annealed case, where interconversion between rod and coil states is possible, the resulting force law is sigmoid with a pronounced plateau. This last case is realized, for example, when homopolypeptides capable of undergoing a helix-coil transition are extended from a coil state. Both scenarios are relevant to the analysis and design of experiments involving single molecule mechanical measurements of biopolymers and synthetic macromolecules.
\end{abstract}

PACS numbers: $61.25 . \mathrm{Hq}, 61.41 .+\mathrm{e}, 87.15 . \mathrm{He}$

With the advent of single molecule mechanical measurements it became possible to study the force laws characterizing the extension of individual macromolecules [1]. In turn, these provide a probe of internal degrees of freedom associated with intrachain self assembly or with monomers that can assume different conformational states. A molecular interpretation of the force laws thus obtained requires appropriate theoretical models allowing for the distinctive "internal states" of each system. The formulation of such models is a challenging task in view of the complexity and diversity of the systems investigated. These include DNA [2], the muscle protein titin [3] and the extracellular matrix protein tenascin [⿰氵⿴囗十), the polyscharides dextran [5] and xanthan [6] as well as the synthetic polymer poly(ethelene-glycol) [7].

In this letter we consider two unexplored yet accessible systems where a detailed confrontation between theory and experiment is possible. In particular, we present a theory for the extension force law of multiblock copolymers consisting of alternating rod and coil blocks. Two scenarios are considered, both focusing on the equilibrium force law of long chains undergoing quasistatic extension. In one, the architecture is "quenched" that is, the block structure is set by the chemistry and no interconversion is possible. Such is the case, for example, for segmented polyurethans [8]. In the second scenario the monomers can interconvert between coil and rod states. The "annealed" architecture is realized, for instance, in homopolypeptides capable of undergoing a cooperative helix-coil transition [9 11]. In this system, the highly rigid helical domains play the part of the rod blocks. As we shall see, the two scenarios lead to distinctive force laws (Figure). The force law of the quenched case is characterized by an abrupt change of slope. This arises because the rod blocks are more susceptible to orientation by the applied tension. In the annealed scenario, the extension of a chain that is initially in a coil state leads to a sigmoid force profile exhibiting a pronounced plateau. The plateau is traceable to a one dimensional coexistence of helical and coil domains where the chain extension favors the helical state because of its low configurational entropy. These results suggest that force measurements can be used to probe the block structure of polymers with quenched architecture. In the annealed case the force law provides a direct measure of the thermodynamic parameters involved. From the view point of the growing field of single molecule mechanical measurements, these results are helpful in exploring the diagnostic potential of these techniques. The discussion is of interest from the polymer physics perspective because the theory of polymer elasticity focuses on the case of flexible homopolymers modeled as random walks, or self avoiding random walks, with a constant step length and no internal degrees of freedom 11. In this context, rod-coil multiblock copolymers with quenched architecture may be viewed as a special case of heteropolymers incorporating monomers of different sizes. The analysis of the annealed case supplements the isolated discussions of the effect of internal degrees of freedom on the extension elasticity 12 15].

We first consider the elastic free energy $F_{e l}$ and the tension $f=\partial F_{e l} / \partial R$ of a quenched rod-coil multiblock copolymer with an imposed end-to-end distance, $R$. The chain incorporates $N$ monomers of identical length $a$ that form $y N$ rod blocks and $y N$ coil blocks such that the number of monomers in the rods is $\theta N$. For simplicity we assume that the coil and rod blocks are monodispersed, and that the number of monomers in a rod block is thus $\theta / y$. We focus on the case of long rod blocks, $\theta / y \gg 1$. The distinctive feature of rod-coil multiblock copolymers is that the rodlike blocks, the longer "monomers", are oriented by the applied tension before the alignment of the shorter monomers becomes significant. The freely jointed chain model, the macromolecular analog of the Langevin's theory of paramagnetism, enables a quantitative description of this situation [12,16]. The length of the monomer is the counterpart of the magnetic moment, the applied tension is the analog of the magnetic field while the end-to-end distance of the chain corresponds to the magnetization. A monomer of length $l$ is assigned an orientational energy of $-f l \cos \phi$ where $\phi$ is the angle made by $\mathbf{l}$ with respect to $\mathbf{f}$. For a flexible homopolymer the end-to-end distance is $R=N a L(x)$ 
where $L(x)=\operatorname{coth} x-x^{-1}$ is the Langevin function and $x=f a / k T$. In our situation the multiblock copolymer is viewed as a collection of two types of non-interacting "dipoles": $(1-\theta) N$ monomers of length $a$ and $y N$ "monomers" of length $\theta a / y$. The reduced end-to-end distance, $r=R / N a$ is thus
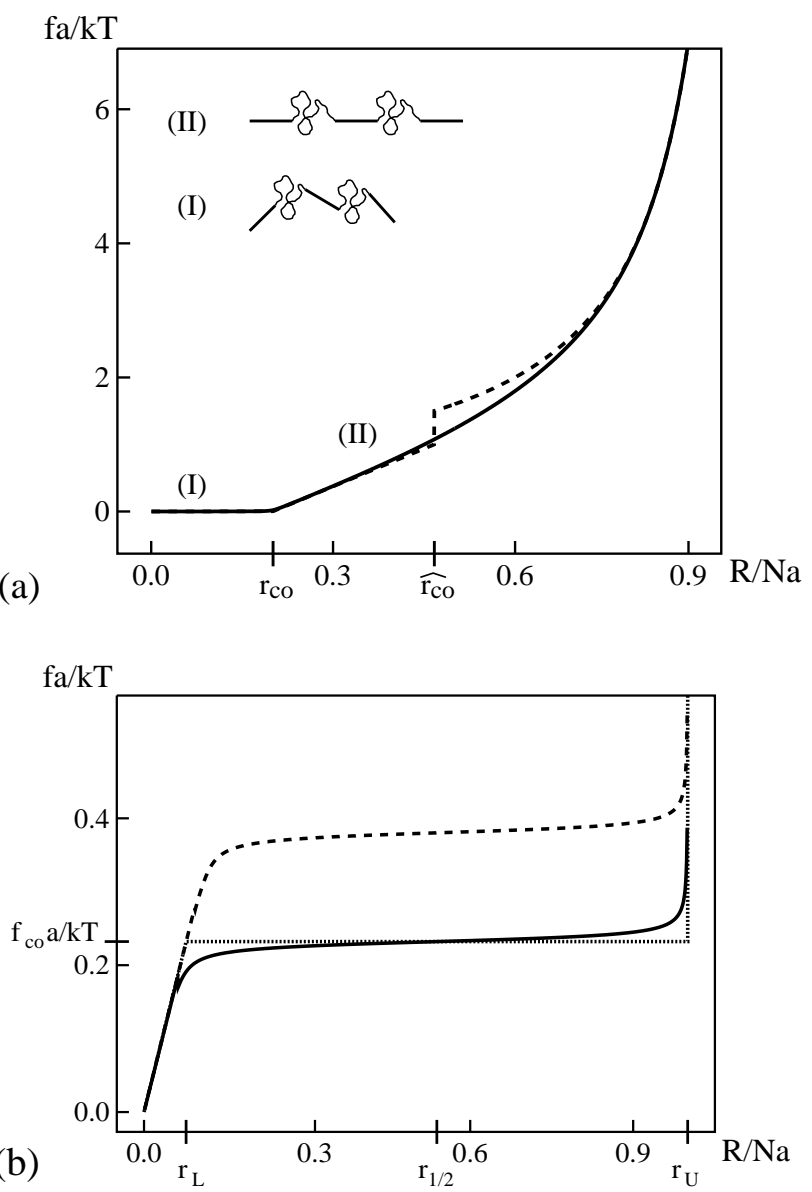

FIG. 1. The force laws characterizing the extension of a rod-coil multiblock copolymer with a quenched (a) and an annealed (b) architectures. The parameters for (a) are $\theta=0.2$ and $y=10^{-4}$. The continuous line depicts the exact force law while the dashed line describes the approximate expression discussed in the text. The chain configurations in regions (I) and (II) are schematically depicted above. The parameters for (b) are $s=0.7$ (dashed line) and $s=0.8$ (continuous line). In both cases $\sigma=10^{-4}$. The dotted line depicts the $S_{m i x}=0$ approximation for $s=0.8 . f_{c o}, r_{L}, r_{1 / 2}$ and $r_{U}$ correspond to $s=0.8$ case.

$$
r=(1-\theta) L(x)+\theta L(\theta x / y) .
$$

Three regimes are involved. When $x \ll 1$ and $\theta x / y \ll 1$ both the rods and the $a$ monomers are weakly oriented. Since $L(z) \approx z / 3$ for $z \ll 1$, we find $\mathrm{fa} / k T \approx$ $3 r /\left(1-\theta+\theta^{2} / y\right)$ and $F_{e l} / N k T \approx 3 r^{2} / 2\left(1-\theta+\theta^{2} / y\right)$. For stronger extensions $x \ll 1$ while $\theta x / y \gg 1$ in other words, the $a$ monomers are only weakly aligned but the rods are almost fully oriented with $f$. Focusing on the case of long rods, $y \ll 1$, when $L(\theta x / y) \approx 1$, we obtain $f a / k T \approx 3(r-\theta) /(1-\theta)$ and $F_{e l} / N k T \approx$ $3(r-\theta)^{2} / 2(1-\theta)+3 y / 2$. The first term in $F_{e l}$ reflects the Gaussian stretching penalty of the $(1-\theta) N$ "short" monomers. It allows for the full alignment of the rods and the resulting modification of the imposed end-to-end distance experienced by the flexible blocks. The second term reflects the Gaussian penalty associated with the full alignment of the $y N$ rod blocks. The crossover between the two regimes occurs at $r_{c o} \approx \theta+y(1-\theta) / \theta$. Eventually, upon further extension, the $a$ monomers also approach saturation, $x \gg 1$ and $\theta x / y \gg 1$. In this last regime $f a / k T \approx(1-\theta+y) /(1-r)$ and $F_{\text {el }} / N k T \approx$ $(1-\theta+y) \ln [2(1-\theta) / 3(1-r)]+3 y / 2+(1-\theta) / 6$. Since the approximate expressions for $f$ do not match at the boundary between the two last regimes, the crossover between them, $\widehat{r_{c o}}$, may be determined by the requirement that $f a / k T \approx 3(r-\theta) /(1-\theta) \approx 1$ leading to $\widehat{r_{c o}} \approx(1+2 \theta) / 3$. Altogether, the plot of $f a / k T$ vs. $r$ for a rod-coil multiblock copolymer is distinguished by an abrupt change of slope at $r_{c o}$. In turn, this feature provides a useful diagnostic for the architecture of the polymer.

In the annealed scenario, monomers may interconvert between the rod and coil states. Accordingly, $\theta$ and $y$ are no longer constants set by the chemistry. Rather, their values vary with the temperature $T$ and with $R$. $\theta$ and $y$ are determined by minimizing the free energy per chain $F_{\text {chain }}$ with respect to $y$ and $\theta$ for a given $R$. $F_{\text {chain }}$ allows, in addition to $F_{e l}$, for the mixing free energy $F_{m i x}$ of the one dimensional mixture, along the chain trajectory, of the rod and coil blocks. Our discussion focuses on polymers undergoing a helix-coil transition. It is directed at the coupling of the helix-coil transition with the extension for the simplest possible situation, that is a homopolypeptide capable of forming an $\alpha$-helix [9 11$]$. This form of intrachain self assembly is due to intrachain hydrogen bonds. The $i$-th monomer, residue, forms $\mathrm{H}$ bonds with the $(i+3)$ and $(i-3)$ monomers. Altogether, a helical domain consisting of $n$ monomers involves $n-2$ H-bonds. The persistence length of an $\alpha$-helix is of order of $200 \mathrm{~nm}$ and a helical domain may thus be considered as a rod block. It is convenient to discuss this system in terms of "bonds" between two adjacent monomers. For simplicity we retain the approximation setting the monomer size in the rod and coil state equal to $a$. Using the coil state as a reference, each helical bond is assigned a free energy $\Delta f$. It reflects the contribution of the intrachain $\mathrm{H}$-bonds, the change in solvation due to the formation of intrachain H-bonds and the associated loss of configurational entropy. The helix-coil transition occurs at the transition temperature $T_{*}$ when $\Delta f=0$. Above $T_{*}, \Delta f>0$ while below $T_{*}, \Delta f<0$. Terminal helical bonds, at the boundary of a helical domain, incur an additional free energy penalty $\Delta f_{t}>0$ since the terminal monomers lose their configurational entropy but do not contribute H-bonds. $\Delta f_{t}$ plays the role of interfacial free energy associated with the helix-coil domain 
boundary. Traditionally, the theory of the helix-coil transition is formulated in terms of the Bragg-Zimm parameters $s=\exp (-\Delta f / k T)$ and $\sigma=\exp \left(-2 \Delta f_{t} / k T\right)$ where $\sigma \approx 10^{-3}-10^{-4}$ varies with the identity of the residues but is independent of $T$. The helix-coil transition gives rise to a sigmoid $\theta$ vs. $T$ plot with a characteristic width $\sim T_{*} \sigma^{1 / 2}$. The usual discussion of the helix-coil transition is based on transfer matrix methods. For our purposes it is convenient to recast it in terms of the free energy of the unperturbed chain, $F_{\text {chain }}^{0}$. For long chain, when the $N \rightarrow \infty$ limit is applicable, $F_{\text {chain }}^{0}$ reflects three contributions. Each of the $\theta N$ helical bonds contributes $\Delta f$ while every one of the $2 y N$ terminal bonds contributes $\Delta f_{t}$. These two terms are supplemented by the mixing entropy, $S_{m i x}$, associated with the different possible combinations of $\theta N$ helical bonds and $2 y N$ terminal bonds. The number of possible configurations, $W$ is $W_{t h} W_{t c}$ where $W_{t h}=\left(\begin{array}{l}\theta N \\ y N\end{array}\right)$ is the number of ways of placing $y N$ terminal bonds among $\theta N$ helical bonds and $W_{t c}=\left(\begin{array}{c}(1-\theta) N \\ y N\end{array}\right)$ is the statistical weight associated with the placement of $y N$ terminal bonds among $(1-\theta) N$ coil bonds. $S_{m i x}=k \ln W$ can be expressed as $S_{m i x}=$ $\theta S_{\text {mix }}(y / \theta)+(1-\theta) S_{\text {mix }}(y /(1-\theta))$ where $\frac{1}{N} S_{\text {mix }}(X)=$ $-X \ln X-(1-X) \ln (1-X)$. Here $\frac{1}{N} S_{\text {mix }}(y / \theta)$ is the mixing entropy of the terminal monomers among the rod monomers while $\frac{1}{N} S_{m i x}(y /(1-\theta))$ is the mixing entropy of the terminal monomers among the coil monomers. Altogether

$$
\begin{aligned}
\frac{F_{\text {chain }}^{o}=}{N k T} & \theta \frac{\Delta f}{k T}+2 y \frac{\Delta f_{t}}{k T}+(\theta-y) \ln \frac{\theta-y}{\theta}+y \ln \frac{y}{\theta} \\
& +(1-\theta-y) \ln \frac{1-\theta-y}{1-\theta}+y \ln \frac{y}{1-\theta} .
\end{aligned}
$$

The equilibrium conditions $\partial F_{\text {chain }}^{0} / \partial y=0$ and $\partial F_{\text {chain }}^{0} / \partial \theta=0$ lead respectively to $y^{2}=(\theta-y)(1-$ $\theta-y) \sigma$ and to $(1-\theta)(\theta-y)=\theta(1-\theta-y) s$. In turn, these yield the familiar results for the unperturbed chain and, in particular, $\theta=\frac{1}{2}+\frac{1}{2} \sqrt{\frac{(s-1)^{2}}{4 \sigma s+(s-1)^{2}}}$.

The full analysis of the coupling of the helix-coil transition with the extension of the chain involves an augmented free energy per chain, allowing for the extension penalty $F_{\text {chain }}=F_{\text {chain }}^{0}+F_{\text {el }}$ [17]. An analytic solution yielding the qualitative features of the force law, as well as the important length and force scales, is possible by setting $S_{m i x}=0$ [14]. Within the $S_{m i x}=0$ approximation the interfacial penalty imposes coalescence of the helical domains leading to the formation of a helix-coil diblock copolymer $(y=0)$. As a result, the helix-coil transition takes place as a first order phase transition. The corresponding free energy is

$$
\frac{F_{\text {chain }}}{N k T} \approx \frac{3(r-\theta)^{2}}{2(1-\theta)}+\theta \frac{\Delta f}{k T} .
$$

The equilibrium condition $\partial F_{\text {chain }} / \partial \theta=0$ for a given $r$ yields $\theta=1-(1-r) / \sqrt{1-2 \Delta f / 3 k T}$. In turn, this defines a critical extension, $r_{L}=1-\sqrt{1-2 \Delta f / 3 k T}$ such that for $r<r_{L}, \theta=0$ and for $r \geq r_{L}, \theta$ is specified by $\theta=\left(r-r_{L}\right) /\left(1-r_{L}\right)$. The equilibrium free energy of the chain for $r<r_{L}$ is $F_{\text {chain }} / N k T \approx 3 r^{2} / 2$ while for $r \geq r_{L}$ it is $F_{\text {chain }} / N k T \approx 3 r_{L}(r-1)+\Delta f / k T$. This regime lasts until $\theta=1$ is attained at $r_{U}=1$. Within this simple minded picture, further extension is impossible and for $r_{U}>1, F_{\text {chain }}=\infty$. The corresponding force law involves three regimes: (i) A linear response regime, where the Gaussian elasticity is operative and $f a / N k T \approx 3 r$, occurs while $r<r_{L}$ and $\theta=0$. (ii) A plateau with $f_{c o} a / N k T \approx 3 r_{L}$ occurs in the range $r_{L}<r<r_{U}$ when the coexistence between the helix and coil states follows the lever rule $\theta /(1-\theta)=\left(r-r_{L}\right) /\left(r_{U}-r\right)$. The midpoint of the plateau, corresponding to $\theta=1 / 2$, occurs at $r_{1 / 2}=\left(r_{U}+r_{L}\right) / 2$. (iii) A steep increase in force occurs at $r=r_{U}$, when the assumed nonextensibility of the fully helical chain comes into play. Note that in reality the helical domains are not perfect rods. High applied tension may cause extension by breaking the H-bonds. Within the $S_{m i x}=0$ approximation the plateau corresponds to a first order phase transition involving the coexistence of a helical phase and a coil phase. Such phase transition is prohibited in one dimensional system experiencing short ranged interactions [18]. When one allows for $S_{m i x}>0$ the plateau in the force law exhibits a weak increase with $r$, instead of the $r^{0}$ dependence predicted by the $S_{m i x}=0$ approximation. Yet, as we shall see, the center of the plateau occurs at $r_{1 / 2}$ and its height at this point is $f_{c o} a / k T \approx 3 r_{L}$. Furthermore, the qualitative $\Delta f$ dependence of both the height and the width of the plateau is retained. In particular, the width of the plateau increases while its height decreases as $\Delta f \rightarrow 0$, or equivalently, as $s \rightarrow 1$. Since at the vicinity of the transition temperature $s \sim\left(T-T_{*}\right) / T_{*}$, these scenarios may be explored by change of $T$.

The complete analysis of the problem in terms $F_{\text {chain }}=F_{\text {chain }}^{0}+F_{\text {el }}$ involves different regimes distinguished by the applicable form of $F_{e l}$, as discussed in the first part of this letter. For weak extensions $F_{e l} / N k T \approx 3 r^{2} / 2\left(1-\theta+\theta^{2} / y\right)$ and the equilibrium conditions $\partial F_{\text {chain }} / \partial \theta=0$ and $\partial F_{\text {chain }} / \partial y=0$ lead to $\partial F_{\text {chain }}^{0} / \partial \theta=-\partial F_{\text {el }} / \partial \theta$ and $\partial F_{\text {chain }}^{0} / \partial y=-\partial F_{\text {el }} / \partial y$. In turn, this leads to $y^{2}=(\theta-y)(1-\theta-y) \sigma K_{\sigma}$ and to $(1-\theta)(\theta-y)=\theta(1-\theta-y) s K_{s}$ where $K_{\sigma}=$ $\exp \left[\left(\partial F_{e l} / \partial y\right) / N k T\right]$ and $K_{s}=\exp \left[\left(\partial F_{e l} / \partial \theta\right) / N k T\right]$. $\left(\partial F_{e l} / \partial \theta\right) / N k T$ is the increment in the elastic free energy per monomer associated with adding one residue to a helical block for a constant $y$ and $\left(\partial F_{e l} / \partial y\right) / N k T$ is the price of creating an extra helical sequence while maintaining a constant $\theta$. For weak extensions, when the elastic penalty is a weak perturbation, $K_{\sigma}$ and $K_{s}$ may be approximated by $K_{\sigma}=\exp \left[-r^{2} \theta_{0}^{2} / y_{0}^{2}\left(1-\theta_{0}+\theta_{0}^{2} / y_{0}\right)^{2}\right]$ and $K_{s}=\exp \left[r^{2}\left(2 \theta_{0} / y_{0}-1\right) /\left(1-\theta_{0}+\theta_{0}^{2} / y_{0}\right)^{2}\right]$ where $\theta_{0}$ and $y_{0}$ correspond to the unperturbed chain. $K_{\sigma}$ is a decreasing function of $r$ while $K_{s}$ is an increasing function of $r$. Thus, the extension favors the transition by increasing $s$ and enhances the cooperatively by decreasing $\sigma$. This analysis demonstrates that the 
$S_{\text {mix }}=0$ approximation overestimates $F_{\text {el }}$ and thus $f$. $f a / N k T \approx 3 r /\left(1-\theta_{0}+\theta_{0}^{2} / y_{0}\right)$ provides a better estimate for $f$. However, in accordance with the Le Chatellier principle [18], the actual force law is even weaker since $\theta$ increases with $r$ while $y / \theta$ decreases. For stronger deformations, when $r>r_{c o}$ the appropriate elastic free energy is $F_{e l} / N k T \approx 3(r-\theta)^{2} / 2(1-\theta)+3 y / 2$. In this range it is possible to obtain the force law at the vicinity of $\theta=1 / 2$. The equilibrium condition $\partial F_{\text {chain }} / \partial y=0$ leads to $y^{2}=(\theta-y)(1-\theta-y) \sigma / e^{3 / 2}$. Since $\sigma \ll 1$ this reduces, in the vicinity of $\theta=1 / 2$, to $y \approx \sqrt{\theta(1-\theta) \sigma / e^{3 / 2}}$. Utilizing this relationship, and following expansion of the logarithmic terms we obtain $F_{\text {chain }} / k T \approx 3(r-$ $\theta)^{2} / 2(1-\theta)+\theta \Delta f / k T-2 \sqrt{\theta(1-\theta) \sigma / e^{3 / 2}}$. In turn, $\partial F_{\text {chain }} / \partial \theta=0$ recovers the expression for $\theta$ as obtained within the $S_{m i x}=0$ approximation, $\theta \approx\left(r-r_{L}\right) /\left(1-r_{L}\right)$. This allows us to express $F_{\text {chain }}$ as a function of $r$ and to obtain $f$ in the vicinity of $\theta=1 / 2$

$$
\frac{f a}{k T} \approx \frac{f_{c o} a}{k T}+2 \frac{\sigma^{1 / 2}}{e^{3 / 4}} \frac{\left(r-r_{1 / 2}\right)\left(1-r_{L}\right)^{-1}}{\sqrt{\left(r-r_{L}\right)\left(r_{U}-r\right)}} .
$$

The force at $r_{1 / 2}$ is $f_{c o}$ as suggested by the $S_{m i x}=0$ approximation. However, when the full $F_{\text {chain }}$ is allowed for the $f \sim r^{0}$ behavior of a perfect plateau is lost. The second term in (14) ensures that $f$ increases with $r$. On the other hand, since $\sigma \approx 10^{-4}$, the $\sigma^{1 / 2}$ prefactor in this term imposes slow variation of $f$ in the vicinity of $r_{1 / 2}$.

Our discussion of the elasticity of rod-coil multiblock copolymers reveals two scenarios. In the quenched case, the force law exhibits a sharp change of slope while in the annealed case the force law is sigmoid. It is important to note that our analysis focused on the thermodynamic force law as obtained for long chains and quasistatic extension. Effects due to the finite length of the chains and the finite rate of deformation may modify the experimentally observed force curves [15]. The analysis of the coupling between the helix-coil transition and the chain extension focused on the case of homopolypeptides capable of forming a single strand $\alpha$-helix. The results can be however applied, with some modifications, to a much wider class of systems. It is relevant, for example, to the interpretation of the sigmoid force curve observed for poly(ethylene-glycol) which was attributed to the formation of water-mediated single stranded helix [7]. Furthermore, the analysis can be extended to the case of polymers forming multistranded helices such as collagen. In this context it is of interest to note that sigmoid force curves were indeed observed in early experiments involving fibers formed by fibrous proteins, collagen, keratin etc., that form helical structures [19]. Finally, one should note the recent development of facile synthesis route for homopolypeptides and block copolypeptides of well defined architecture [20]. This provides a convenient method for synthesizing rod-coil multiblock copolymers with either quenched or annealed architecture.
[1] A. D. Mehta et al., Science, 283, 1689 (1999)

[2] R. H. Austin et al., Physics Today 50(2), 32 (1997)

[3] (a) Kellermayer et al., Science 276, 1112 (1997) (b) L. Tskhovrebova et al., Nature 387, 308 (1997) (c) M. Rief et al., Science 276, 1109 (1997)

[4] A. F. Oberhauser et al., Nature 393, 181 (1998)

[5] M. Rief et al., Science 275, 1295 (1997)

[6] H. Li et al., Adv. Mater. 3(4), 316 (1998)

[7] F. Oesterhelt, M. Rief and H. E. Gaub, New J. Phys. 1, 6.1-6.11 (1999)

[8] U. Eisele, Introduction to Polymer Physics (Springer Verlag, Berlin, 1990)

[9] T. M. Birshtein and O. B. Ptitsyn, Conformations of Macromolecules (John Wiley, New York, 1966)

[10] D. Poland and H. Scheraga, Theory of Helix-Coil Transitions in Biopolymers (Academic Press, New York, 1970)

[11] A. Yu. Grosberg and A. R. Khokhlov, Statistical Physics of Macromolecules (AIP Press, New York, 1994)

[12] T. L. Hill, An Introduction to Statistical Thermodynamics (Addison-Wesley, Reading, 1960)

[13] P. Cluzel et al., Science, 271, 792 (1996)

[14] O.V. Borisov and A. Halperin, Europhys. Lett. 34, 657 (1996), Phys. Rev. E 57, 812 (1998), Euro. Phys. J. B 9, 251 (1999)

[15] M. Rief, J. Fernandez and H. E. Gaub, Phys. Rev. Lett. 81, 4764 (1998)

[16] By adopting the freely jointed chain model we ignore possible effects due to the self avoidance of the chain, that is the Pincus elasticity.

[17] Within this model, the derivation of $F_{e l}$ and $S_{m i x}$ involve different assumptions. $F_{e l}$ is obtained for monodispersed blocks while $S_{m i x}$ reflects the polydispersity of the domains.

[18] L. D. Landau and E. M. Lifshitz, Statistical Physics (Pergamon, Oxford, 1980)

[19] P. J. Flory, Science 124, 53 (1956)

[20] T. J. Deming, Nature, 390, 386 (1997) 